\title{
The relation between cerebral oxygenation and lactate in infants with hypoxic-ischemic encephalopathy treated with hypothermia
}

\author{
Leanne Erkelens-de Vetten, Willemien S. Kalteren, Hendrik J. ter Horst, \\ Arend F. Bos, Elisabeth M.W. Kooi
}

Division of Neonatology, Beatrix Children's Hospital, University Medical Center Groningen, the Netherlands Authors have nothing to disclose

\section{Background}

In infants with moderate to severe hypoxicischemic encephalopathy (HIE), blood circulation is often compromised. Besides serum lactate, regional cerebral oxygen saturation $\left(\mathrm{rCSO}_{2}\right)$ may be a marker for hemodynamic status. A (negative) relation between these two markers, may support the effect of hemodynamic status on both.

\section{Aim}

We aimed to examine during the first 4 days of life, the cross-sectional relation between $\mathrm{rCSO}_{2}$ and serum lactate, in cooled infants with HIE with and without inotrope support.

\section{Methods}

- Retrospective cohort study.

- Inclusion: all cooled infants with cerebral NIRS measurements from 2010-2016 in a single centre tertiary NICU.

- Correlation of lactate levels and averaged $\mathrm{rcSO}_{2}$ values measured during periods between $6,12,24$, 48,72 , and 96 hours after birth.

- For the entire group, and in the subgroups of infants treated with inotropes.

\section{Results}

Seventy-three infants were included in this study. Patient characteristics are shown in table 1.

\begin{tabular}{|c|c|}
\hline$N=73$ & \\
\hline Birth weight (gram) & 3350 (1760-4990) \\
\hline Gestational age (weeks) & $40(36-42)$ \\
\hline 5' Apgar Score & $3(0-7)$ \\
\hline 1st lactate & $17.5(1.9-71)$ \\
\hline
\end{tabular}

- Twenty-one infants received inotropes.

- Before $72 \mathrm{hrs}$, we found no significant correlation coefficients between $\mathrm{rCSO}_{2}$ and lactate (shown in table 2$)$. Only at $72 \mathrm{hrs}$, they correlated $(\rho=$ $+0.33, p=0.011$ ).

- Within the subgroup of infants that received inotropes no significant correlations were found at any time point.

- Of note, lactate, but not $\mathrm{rcSO}_{2}$, was persistently higher in the inotrope treated subgroup, compared to the infants without inotropes.

\begin{tabular}{lllllll} 
& $0-6 \mathrm{hr}$ & $6-12 \mathrm{hr}$ & $12-24 \mathrm{hr}$ & $24-48 \mathrm{hr}$ & $48-72 \mathrm{hr} *$ & $72-96 \mathrm{hr}$ \\
\hline $\begin{array}{l}\text { Median } \\
\text { (IQR)Lactate } \\
\text { (mmol/L) }\end{array}$ & $13.5(7.3-17.5)$ & $7.6(4.3-12.3)$ & $4.4(3.2-9.4)$ & $2.9(2.1-5.1)$ & $1.8(1.5-2.5)$ & $1.3(1.0-2.0)$ \\
$\begin{array}{l}\text { Median } \\
\text { (IQR)RcSO }\end{array}$ & $71(61-78)$ & $72(66-77)$ & $79(74-84)$ & $87(81-91)$ & $88(83-90)$ & $86(80-89)$ \\
\hline
\end{tabular}

Table 2. Course of lactate and rcSO2 values total group (IQR-interquartile range). * Statistical significant correlation between lactate and rcSO2.

\section{Conclusions}

Although a higher lactate and the use of inotropes does not necessarily reflect poorer hemodynamics, these results may suggest that rcSO2 is a poor marker for hemodynamic status in HIE infants, possibly due to the decreased cerebral oxygen demand due to HIE, hypothermia and sedation. 